\title{
Drug allergy is Associated with the Development of Extraintestinal Manifestations in Patients with Ulcerative Colitis
}

${ }^{1}$ Inflammatory Bowel Disease Clinic, Department of Gastroenterology, Instituto Nacional de Ciencias Médicas y Nutrición Salvador Zubirán, Mexico City, Mexico

${ }^{2}$ Programa de Estudios Combinados en Medicina (PECEM), Facultad de Medicina, Universidad Nacional Autónoma de México (UNAM), Mexico City, Mexico

\section{KeY WorDs}

drug allergy; ulcerative colitis; extraintestinal manifestations; IBD pathogenesis

\section{Corresponding author}

Jesús K. Yamamoto-Furusho

IBD Clinic, Department of Gastroenterology

Instituto Nacional de Ciencias Médicas y Nutrición

Salvador Zubirán

Vasco de Quiroga 15, Colonia Belisario Domínguez

Sección XVI, Tlalpan, CP 14080,

Mexico City, Mexico

Phone: +525555733418

Fax: +5255 56550942

E-mail: kazuofurusho@hotmail.com

\section{Doi}

10.23822/EurAnnACI.1764-1489.110

\begin{abstract}
Summary
Drug allergies are developed by antibody or cell-mediated reactions as immunologic mechanisms. It has been demonstrated that hypersensitivity reaction to certain allergens may play a role in the pathogenesis of inflammatory bowel disease (IBD) focused on food allergies.

A total of 256 patients with UC were divided in two groups: 203 patients with active UC and 53 in remission UC were included in the present study. In the present study we found that $11.7 \%$ had allergy to at least one drug distributed. The most frequent drug-allergies were sulfonamides in $2.8 \%$ and penicillin in $3.1 \%$. Sulfonamide allergy was associated with several extraintestinal manifestations such as: peripheral arthritis / arthralgia $(O R=9.06,95 \%$ $C I 1.71-48.00, p=0.002)$; pyoderma gangrenosum $(O R=24.10,95 \% C I$ $3.55-163.48, p<0.0001)$ and uveitis $(O R=15.93,95 \% C I 2.55-99.23$, $p<0.0001$ ). The frequency of drug allergy was $11.7 \%$ in Mexican UC patients, most frequently to sulfonamides and penicillin drugs. The presence of sulfonamide allergy was associated with the presence of several extra-intestinal manifestations.
\end{abstract}

\section{Introduction}

Several gene mutations that control innate immune recognition, adaptive immunity, and epithelial permeability are associated with gut inflammation. These phenomena suggest that perturbations of homeostasis between gut antigens and host immunity represent a critical determinant in the development of gut inflammation and allergy (1). An abnormal intestinal permeability is one of the hallmarks of an inflamed gut that has been observed in around $42.9 \%$ of patients affected by Ulcerative Colitis (UC) (2).

Intestinal barrier defects have been associated with a broad range of diseases, including GI (celiac disease, inflammatory bowel disease and colon carcinoma) but also extra intestinal disorders (chronic liver disease, type 1 diabetes, obesity) (3).
A previous study reported that UC patients may have an allergic etiology in approximately 66\%; several cases of UC have been associated with pollen allergy milk consumption and allergy to other specific foods $(4,5)$. In previous studies where specific immunotherapy was administered, it was demonstrated that alterations in regulation of IgE, Il4, TNF- $\alpha$ and IgG4 had an important involvement in the pathogenesis of Inflammatory Bowel Disease (IBD) (6).

Drug allergies are developed by antibody or cell-mediated reactions as immunologic mechanisms (7). In several cases, the mechanism involved in the development of drug hypersensitivity is mediated by T-cells (Type IV hypersensitivity) and is associated with a wide range of clinical manifestations, ranging from affection of skin to anaphylaxis. Frequently drugs involved in this type of allergic mechanism are sulfa antibiotics and $\beta$-lac- 
tams (8). A Th2 response is characteristic of the subject with allergy and it is also present in patients with UC (9).

Also, it has been demonstrated that hypersensitivity reaction to certain allergens may play a role in the pathogenesis of inflammatory bowel disease (IBD) focused on food allergies. Even specific immunotherapy has demonstrated improvement in the clinical course of patients with UC (10).

No previous studies have evaluated the role of drug allergy in patients with UC. The aim of the present study was to determine the most frequent drug allergies in Mexican patients with $\mathrm{UC}$ and its association with clinical outcomes.

\section{Materials and methods}

A total of 289 patients with histopathologic diagnosis of UC belonging to the Inflammatory Bowel Disease Clinic at the Instituto Nacional de Ciencias Médicas y Nutrición Salvador Zubirán were recruited in the period between 2016 and 2017. Sociodemographic variables (current age, gender, occupation), clinical (age at diagnosis, smoking habit, appendectomy, clinical course of disease, extent according to Montreal's classification, extraintestinal manifestations, concomitant autoimmune diseases, number of previous hospitalizations, disease activity by endoscopic and histologic according to Mayo's and Riley's classification respectively, and current medical treatment. All previous or current medication allergy were documented by frequency such as penicillin, sulpha drugs, acetylsalicylic acid, metoclopramide, iodeine, ceftriaxone, metamizole, quinolones, pseudoephedrine, cefuroxime, acetaminophen, naproxen, ambroxol, opioids, infliximab, racecadotril, thiethylperazine.

\section{Statistical analysis}

The Kolmogorov-Smirnov test was used as normality test. Descriptive statistics as well as Chi squared and Fisher's exact test for categorical variables and U-Mann Whitney or $\mathrm{t}$ Student test for numerical variables. The strength of the association was determined by Odds Ratio (OR) and $\mathrm{p}<0.05$ was considered as statistically significant. All data was analyzed by SPSS v. 24 statistical analysis program.

\section{Results}

A total of 256 patients with UC were divided in two groups: 203 patients with active UC and 53 in remission UC were included in the present study. A 51\% ( $\mathrm{n}=130)$ were women and $49 \%$ (n =126) men, with a median age of 41 years (range 18-89 years). The extent of UC was evaluated by colonoscopy in all patients and classified according to Montreal classification, which was distributed as follows: $18.5 \%$ distal colitis; $12.14 \%$ left-sided colitis and $69.36 \%$ extensive colitis. The clinical course of disease was: $46.1 \%$ had initially active and then inactive disease;
$46.1 \%$ showed intermittent disease $(<2$ relapses per year) and $7.8 \%$ continuous disease. In $28.9 \%$ had at least one extra-intestinal manifestation such as arthritis / arthralgia in $22.7 \%$; spondylitis $1.2 \%$; sacroiliitis $1.6 \%$; primary sclerosing cholangitis $6.3 \%$; pyoderma gangrenosum $2.3 \%$, erythema nodosum $0.4 \%$ and uveitis in $3.1 \%$. In only $3.5 \%$ had at least one concomitant autoimmune disease such as: ankylosing spondylitis $0.4 \%$, autoimmune hepatitis $1.2 \%$, systemic lupus erythematosus $0.4 \%$, idiopathic thrombocytopenic purpura $0.4 \%$, Hashimoto's thyroiditis $0.4 \%$, multiple sclerosis $0.4 \%$ and vitiligo $1.2 \%$.

In the present study, we found that $11.7 \%$ had allergy to at least one drug distributed as shown in figure $\mathbf{1}$. The most frequent drug-allergies were penicillin in $3.1 \%$, sulfonamides in $2.8 \%$, acetylsalicylic acid $1 \%$ and other drugs under $1 \%$.

\section{Association between drug allergy and clinical outcomes}

Sulfonamide allergy was associated with several extraintestinal manifestations such as: peripheral arthritis / arthralgia (OR = 9.06, 95\% CI $1.71-48.00, \mathrm{p}=0.002$ ); pyoderma gangrenosum $(\mathrm{OR}=24.10,95 \% \mathrm{CI} 3.55-163.48, \mathrm{p}<0.0001)$ and uveitis $(\mathrm{OR}=15.93,95 \%$ CI $2.55-99.23, \mathrm{p}<0.0001)$.

\section{Discussion}

In the present study, we found that the most common drug-allergies were penicillin in $3.1 \%$, sulfonamides in $2.8 \%$, acetylsalicylic acid $1 \%$, and other drugs under $1 \%$. Our results contrast from cross-sectional survey of a general adult population of Portugal, that found a 7.8\% prevalence of self-reported drug allergy, of which $4.5 \%$ were to penicillins or other b-lactams, $1.9 \%$ to aspirin or other NSAIDs, and $1.5 \%$ to other drugs (11). Important to note in our study, the presence of allergy to sulfonamides was associated with extra-intestinal manifestations in Mexican UC patients.

Hypersensitivity reactions represent about one third of all adverse drug reactions. Adverse drug reactions affect $10-20 \%$ of hospitalized patients and more than $7 \%$ of the general population (12). A meta-analysis of prospective studies demonstrated an overall incidence of adverse drug reactions of $6.7 \%(95 \%$ CI $5.2-8.2 \%)$. However, it is important to mention that several bias factors might influenced by length of stay, gender and ward type (13).

On the other hand, the presence of food allergy in UC patients $(30.17 \%)$ was higher than general population $(2.34 \%)$ considering the treatment of food allergy treatment is the allergen specific immunotherapy (SIT) and Clostridium butyricum (CB) efficiently inhibited the clinical symptoms of IBD patients with food allergy (14).

Certain specific allergens have been associated with a higher risk of developing UC such as Cow's milk (15). Also, high titers of antibodies had been related with a more symptomatic 
Figure 1 - Frequency of drug allergies in UC patients.

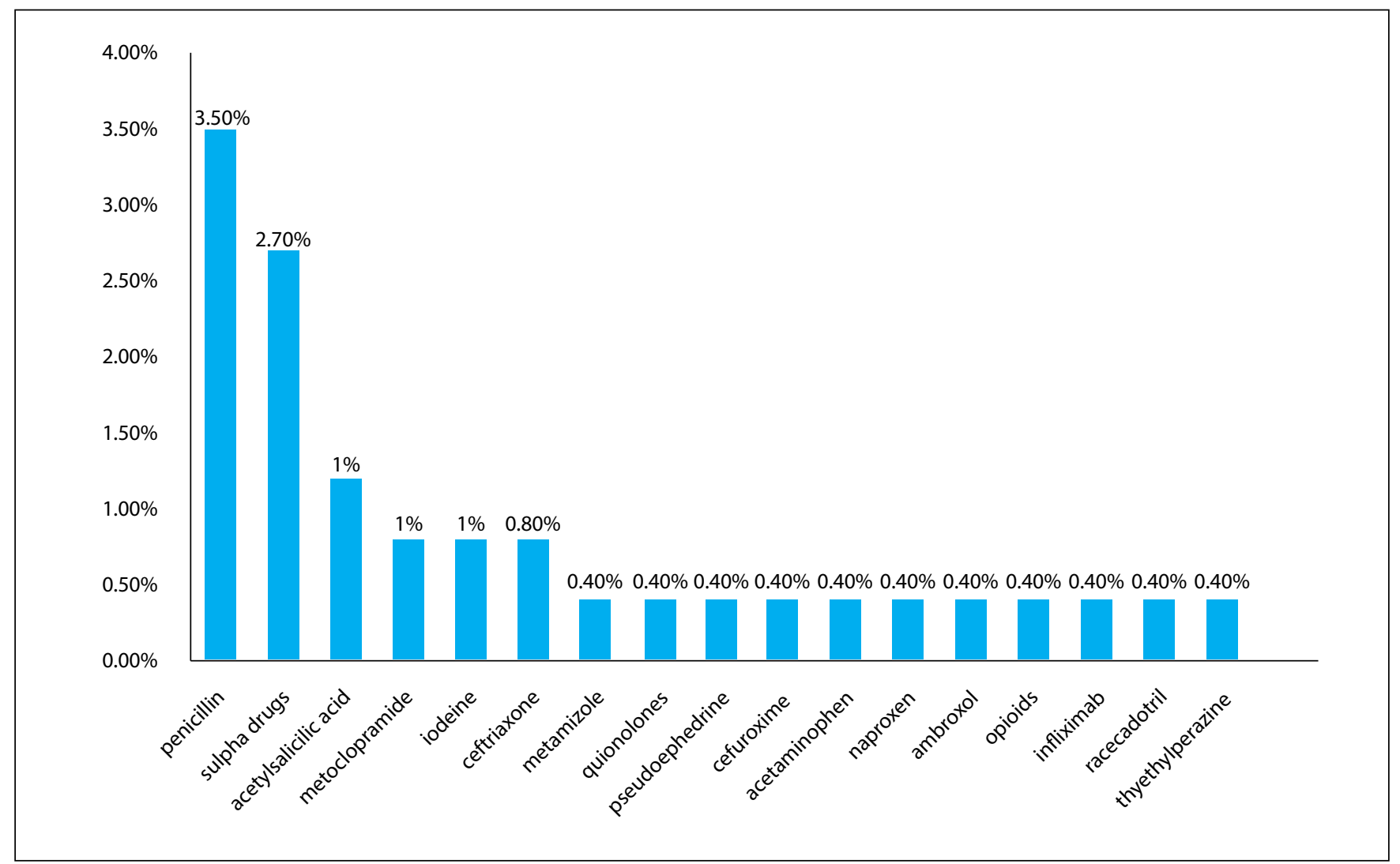

clinical course in the case of subclinical allergy to several foods, most commonly cow's milk (16). Although it has been postulated that allergy could be involved in the development of UC, however, there are no studies about the role of drug allergies in the development of extraintestinal manifestations in patients with UC.

We found an association between the existence of sulfonamide drug allergy and the presence of extraintestinal manifestations such as arthritis / arthralgia, pyoderma gangrenosum and uveitis. This could be explained by a hapten like mechanism, or overlapping antibody-binding sites that predisposes patients with UC to develop affection in sites like joints, skin and uvea (16-22).

Variations in T-cell receptor and both HLA types I and II are more frequent in this population, which could help to explain the propensity of these patients to develop specific extraintestinal manifestations $(23,24)$. On the other hand, regulatory B-cells might have an important role in the development of the extraintestinal manifestations, as postulated in previous studies involving specific immunotherapy (25). Specific immunotherapy has been proposed as a useful alternative for treating the extraintestinal manifestations in patients with uveitis and UC $(11,26)$.

Another hypothesis about the causal link between uveitis and sulfonamide consumption could be that these manifestations are due to a direct effect of such drugs and not to the systemic inflammatory response induced in patients with UC $(27,28)$. Finally, for a better characterization of the systemic inflammatory response of UC patients, it could be useful to analyze which appear first, the reported extraintestinal manifestations or the intestinal disease. Further studies are needed to evaluate the implication of Th2 immunologic response in these patients.

\section{Conclusions}

The frequency of drug allergy was $11.7 \%$ in Mexican UC patients, most frequently to sulfonamides and penicillin drugs. The presence of sulfonamide allergy was associated with the presence of several extraintestinal manifestations such as arthritis / arthralgia, pyoderma gangrenosum and uveitis in Mexican patients with UC. 


\section{Conflicts of interests}

The authors declare they have no conflict of interests.

\section{References}

1. MacDonald TT, Monteleone G. Immunity, Inflammation and Allergy in the Gut. Science 2005; 307(5717):1920-1925.

2. Buning C, Geissler N, Prager M, Sturm A, Baumgart DC, Buttner J, Buhner S, Haas V, Lochs H. Increased intestinal permeability in ulcerative colitis: rather genetic than environmental and a risk factor for extensive disease. Inflamm Bowel Dis 2012; 18(10):19321939.

3. Vancamelbeke M, Vermeire S. The intestinal barrier: a fundamental role in health and disease. Expert Rev Gastroenterol Hepatol 2017; 11(9):821-834.

4. Peña AS, Crusius JB. Food allergy, coeliac disease and chronic inflammatory bowel disease in man. Vet Q 1998; 20Suppl3:S49-52.

5. Cai M, Zeng L, Li LJ, Mo LH, Xie RD, Feng BS, Zheng PY, Liu ZG, Liu ZJ, Yang PC. Allergy Asthma Clin Immunol 2016; 12:37.

6. Johansson SG, Bieber T, Dahl R, Friedmann PS, Lanier BQ, Lockey RF, Motala C, Ortega Martell JA, Platts-Mills TA, Ring J, Thien F, Van Cauwenberge P, Williams HC. Revised nomenclature for allergy for global use: Report of the Nomenclature Review Committee of the World Allergy Organization, October 2003. J Allergy Clin Immunol 2004; 113(5):832.

7. Schnyder B, Pichier WJ. Mechanisms of drug-induced allergy. Mayo Clin Proc 2009; 84(3):268-272.

8. Bamias G, Cominelli F. Role of Th2 immunity in intestinal inflammation. Current opinion in gastroenterology 2015; 31(6):471-476.

9. Cai M, Zeng L, Li LJ, Mo LH, Xie RD, Feng BS, Zheng PY, Liu ZG, Liu ZJ, Yang PC. Specific immunotherapy ameliorates ulcerative colitis. Allergy Asthma Clin Immunol 2016; 12:37.

10. Rowe AH, Rowe A Jr. Chronic ulcerative colitis, atopic allergy in its etiology. Am J Gastroenterol 1960; 34:49-66.

11. Falcao H, Lunet N, Gomes E, et al. Drug allergy in university students from Porto, Portugal. Allergy 2003; 58:1210.

12. Gomes ER, Demoly P. Epidemiology of hypersensitivity drug reactions. Curr Opin Allergy Clin Immunol 2005; 5(4):309-316.

13. Lazarou J, Pomeranz BH, Corey PN. Incidence of adverse drug reactions in hospitalized patients: a meta-analysis of prospective studies. JAMA 1998; 279(15):1200-1205.

14. Demoly P, Bousquet J. Epidemiology of drug allergy. Curr Opin Allergy Clin Immunol 2001; 1(4):305-310.
15. Virta LJ, Ashom M, Kolho KL. Cow's milk allergy, asthma and pediatric IBD. J Pediatr Gastroenterol Nutr 2013; 56(6):649-651.

16. Arezo J, Mohamadreza H, Atefe Yousefi, Mohamad Reza Havasian, Jafar P. Evaluation of dairy allergy among ulcerative colitis patients. Bioinformation 2014; 10(11):693-696.

17. D’Arienzo A, Manguso F, Astarita C, D'Armiento FP, Scarpa R, Gargano D, Scaglione G, Vicinanza G, Bennato R, Mazzacca G. Allergy and mucosal eoshinophil infíltrate in ulcerative colitis. Scand J Gastroenterol 2000; 35(6):624-631.

18. London NJ, Garg SJ, Moorthy RS, Cunningham ET. Drug-induced uveitis. Journal of Ophtalmic Inflammation and Infection 2013; 3:43.

19. Schnyder B, Pichler W. Mechanisms of Drug-Induced Allergy. Mayo Clin Proc 2009; 84(3):268-272.

20. Erkes D, Selvan S. Hapten-Induced Contact Hypersensitivity, Autoimmune Reactions, and Tumor Regression: Plausibility of Mediating Antitumor Immunity. J Immunol Res 2014; 2014:175265.

21. Weltzien HU, Moulon C, Martin S, Padovan E, Hartmann U, Kohler J. T-cell immune responses to haptens. Structural models for allergic and autoimmune reactions. Toxicology 1996; 107(2):141-151.

22. Liao H-Y, Tao L, Zhao J, et al. Clostridium butyricum in combination with specific immunotherapy converts antigen-specific $B$ cells to regulatory B cells in Asthmatic Patients. Scientific Reports 6(2016): 20481.

23. Faulkner L, Meng X, Park BK, Naisbitt DJ. The importance of hapten-protein complex formation in the development of drug allergy. Curr Opin Allergy Clin Immunol 2014; 14(4):293-300.

24. Cameron DJ, Erlanger BF. Evidence for multispecificity of antibody molecules. Nature 1977; 268(5622):763-765.

25. Fan W-L, Shiao M-S, Hui RC-Y et al. HLA Association with Drug-Induced Adverse Reactions. Journal of Immunology Research 2017; 2017:3186328.

26. Howel VM, Holgate ST. HLA genetics and allergic disease. Thorax 1995; 50(8): 815-818.

27. Chen CB, Abe R, Pan RY, Wang CW, Hung SI, Tsai YG, Chung WH. An Updated Review of the Molecular Mechanisms in Drug Hypersensitivity. J Immunol Res 2018; 2018:6431694.

28. Lan B, Yang F, Lu D, Lin Z. Specific immunotherapy plus Clostridium butyricum alleviates ulcerative colitis in patients with food allergy. Sci Rep 2016; 6:25587.

29. Tilden ME, Rosenbaum JT, Fraunfelder FT. Systemic sulfonamides as a cause of bilateral, anterior uveitis. Arch Ophtalmol 1991; 109(1):67-69. 